\title{
Ferroelectricity, Piezoelectricity, and Dielectricity of 0.06PMnN-0.94PZT(45/55) Thin Film on Silicon Substrate
}

\author{
Tao Zhang, ${ }^{1,2}$ Hailong Li, ${ }^{1}$ Chenlei Zhou, ${ }^{1}$ Huaze Zhu, ${ }^{1}$ Yahong Zhou, ${ }^{1}$ Fujun Liang, \\ Huafeng Pang, ${ }^{1}$ Limei Hao, ${ }^{1}$ and Shaorong Li ${ }^{1}$ \\ ${ }^{1}$ College of Science, Xian University of Science and Technology, Xi'an 710054, China \\ ${ }^{2}$ Lab of Modern Acoustics, Institute of Acoustics, Nanjing University, Nanjing 210093, China \\ Correspondence should be addressed to Tao Zhang; tzhang_psu@yahoo.com
}

Received 12 September 2015; Accepted 11 October 2015

Academic Editor: Jun Chen

Copyright (C) 2015 Tao Zhang et al. This is an open access article distributed under the Creative Commons Attribution License, which permits unrestricted use, distribution, and reproduction in any medium, provided the original work is properly cited.

\begin{abstract}
The high piezoelectricity and high quality factor ferroelectric thin films are important for electromechanical applications especially the micro electromechanical system (MEMS). The ternary compound ferroelectric thin films $0.06 \mathrm{~Pb}\left(\mathrm{Mn}_{1 / 3}, \mathrm{Nb}_{2 / 3}\right) \mathrm{O}_{3}$ $+0.94 \mathrm{~Pb}\left(\mathrm{Zr}_{0.45}, \mathrm{Ti}_{0.55}\right) \mathrm{O}_{3}(0.06 \mathrm{PMnN}-0.94 \mathrm{PZT}(45 / 55))$ were deposited on silicon(100) substrates by RF magnetron sputtering method considering that $\mathrm{Mn}$ and $\mathrm{Nb}$ doping will improve PZT properties in this research. For comparison, nondoped PZT(45/55) films were also deposited. The results show that both of thin films show polycrystal structures with the main (111) and (101) orientations. The transverse piezoelectric coefficients are $e_{31, e f f}=-4.03 \mathrm{C} / \mathrm{m}^{2}$ and $e_{31, e f f}=-3.5 \mathrm{C} / \mathrm{m}^{2}$, respectively. These thin films exhibit classical ferroelectricity, in which the coercive electric field intensities are $2 E_{c}=147.31 \mathrm{kV} / \mathrm{cm}$ and $2 E_{c}=135.44 \mathrm{kV} / \mathrm{cm}$, and the saturation polarization $P_{s}=30.86 \mu \mathrm{C} / \mathrm{cm}^{2}$ and $P_{s}=17.74 \mu \mathrm{C} / \mathrm{cm}^{2}$, and the remnant polarization $P_{r}=20.44 \mu \mathrm{C} / \mathrm{cm}^{2}$ and $P_{r}=9.87 \mu \mathrm{C} / \mathrm{cm}^{2}$, respectively. Moreover, the dielectric constants and loss are $\varepsilon_{r}=681$ and $D=5 \%$ and $\varepsilon_{r}=537$ and $D=4.3 \%$, respectively. In conclusion, $0.06 \mathrm{PMnN}-0.94 \mathrm{PZT}(45 / 55)$ thin films act better than nondoped films, even though their dielectric constants are higher. Their excellent ferroelectricity, piezoelectricity, and high power and energy storage property, especially the easy fabrication, integration realizable, and potentially high quality factor, make this kind of thin films available for the realistic applications.
\end{abstract}

\section{Introduction}

The excellent ferroelectric and piezoelectric thin films are the pursuing points for micro-electronic engineers and functional material researchers with the demand of high accuracy, high integration, and high stability in electronic industry, such as the applications for MEMS, FBAR (Thin Film Bulk Acoustic Resonator), actuators, sensors, and FeRAM devices [1-5]. The $\mathrm{Pb}\left(\mathrm{Zr}_{x}, \mathrm{Ti}_{1-x}\right) \mathrm{O}_{3}(\mathrm{PZT})$ based materials own excellent ferroelectricity and piezoelectricity, and so PZT based thin films are intensively studied [6-11]. However, the ferroelectric thin films generally have poor quality factors which are very important parameters for the piezoelectric applications as well $[2,3]$. Some papers reported that the FBARs made of PZT films show very low $Q_{m}$ with the maximum value of 237, and most of them are few tens $[2,3]$, and many researchers have been contributing to various doping and multicompounds compositional PZT based ferroelectric materials for modifying the properties of films by introducing some metals elements to improve the film quality and other properties [8-11].

We got the excellent single crystal PZT(45/55) thin films on $\mathrm{MgO}(001)$ substrate with $6 \% \mathrm{~mol}$ ratio $\mathrm{Pb}\left(\mathrm{Mn}_{1 / 3}\right.$, $\left.\mathrm{Nb}_{2 / 3}\right) \mathrm{O}_{3}(\mathrm{PMnN})$ doped [8], and that kind of thin film owns remarkable properties and beautiful behaviors. To be frank, it is difficult to obtain the perovskite phase of multicompounds PZT based thin films. However, the perfect crystal lattice match between $\mathrm{MgO}$ and PZT acts as the important role in the fabrication. But $\mathrm{MgO}$ substrate also makes the realistic application limited because of the easy chemical etching and nonintegration. As the further work, $0.06 \mathrm{PMnN}$ $0.94 \mathrm{PZT}(45 / 55)$ thin film was fabricated on silicon(100) 
substrate and characterized, and what we have been trying to do is to get the favorable $0.06 \mathrm{PMnN}-0.94 \mathrm{PZT}(45 / 55)$ thin films for the realistic application on silicon substrates.

In this paper, radio frequency (RF) magnetron sputtering method is used to deposit the ternary compound $0.06 \mathrm{PMnN}$ 0.94PZT(45/55) ferroelectric films and nondoped $0.94 \mathrm{PZT}(45 / 55)$ thin films on the $\mathrm{SrRuO}_{3} / \mathrm{Pt} / \mathrm{Ti} / \mathrm{Si}(100)$ substrates, and the quench processing was used for the postheating treatment, and the XRD characterization was made to detect the orientations and the structures of thin films, and the laser vibration detector was used to measure the piezoelectricity $[8,9,12]$, and the Sawyer-Tower circuit was used to test the $P-E$ hysteretic behavior, and L-C-R meter was used to measure the dielectricity $[8,9]$. All the above results were contrasted with each other and also contrasted with the single crystal thin films on $\mathrm{MgO}$ substrates [8], and the general evaluations were given.

\section{Experiment}

The ternary compounds ferroelectric thin film with the composition ratio of $0.06 \mathrm{~Pb}\left(\mathrm{Mn}_{1 / 3}, \mathrm{Nb}_{2 / 3}\right) \mathrm{O}_{3}+0.42 \mathrm{PbZrO}_{3}$ $+0.52 \mathrm{PbTiO}_{3}$ was fabricated on single crystal silicon(100) substrate by RF magnetron sputtering system, and the powder target was made of $\mathrm{PbO}, \mathrm{ZrO}_{2}, \mathrm{TiO}_{2}, \mathrm{MnO}_{2}$, and $\mathrm{Nb}_{2} \mathrm{O}_{5}$ mixed metal oxides powder with enough mixture. Excess $\mathrm{PbO}$ was added into powder target because of the $\mathrm{Pb}$ loss during the high temperature deposition, which is also beneficial for better lattice match between the films and the substrates $[8,9]$. The $\mathrm{Si}(100)$ substrates were prepared with the $\mathrm{SrRuO}_{3}$ buffer layer on $\mathrm{Pt} / \mathrm{Ti}$ bottom electrode, and the sputtering gases are argon (Ar) and oxygen $\left(\mathrm{O}_{2}\right)$ mixture with the ratio of $20: 1$, and the depositing pressure is $1 \mathrm{~Pa}$ with the basis vacuum pressure smaller than $5 \times 10^{-3} \mathrm{~Pa}$. The half an hour presputtering process is always done before each deposition for polishing the surface and excluding the residual moisture of the targets. The sputtering power is $80 \mathrm{w}$, and the substrates were heated up and maintained at $600^{\circ} \mathrm{C}$, and the quenching processes in air are always done after every deposition with the temperature decreasing ratio of $120^{\circ} \mathrm{C} / \mathrm{min}$ during the initial 3 minutes and then they are left to be cool in the room temperature naturally. The deposition rate is about $3-4 \mathrm{~nm} / \mathrm{min}$. All the sputtering conditions are given in detail in Table 1.

The bulk material properties with different mixing ratio of PMnN + PZ + PT ternary compounds are shown in Figure 1. Figures 1(a), 1(b), and 1(c) are the crystal phase, the mechanical quality factor, and the electromechanical coupling coefficient distribution with various ratio of composition, respectively, in which the asterisk "a" represents the ternary compounds composition used in our experiments, and the asterisk " $b$ " represents the classical composition of pure PZT(52/48), which was marked for comparing [8]. It can be obtained that the composition of $0.06 \mathrm{PMnN}$ $0.94 \mathrm{PZT}(45 / 55)$ we used is near the MPB between tetragonal and rhombohedral phase which is also in the area of mechanical quality factor $(Q)$ around 3000, and the electromechanical coupling coefficient $\left(k_{t}\right)$ is predicted to be about 0.40 .
TABLE 1: The conditions of sputtering.

\begin{tabular}{lc}
\hline Target & $\mathrm{PMnN}+\mathrm{PZT}(45 / 55), \mathrm{PZT}(45 / 55)$ \\
Substrate & $\mathrm{SRO} / \mathrm{Pt} / \mathrm{Ti} / \mathrm{Si}(100)$ \\
Gas & $\mathrm{O}_{2}: \mathrm{Ar}=1: 20$ \\
Growth temperature & $600^{\circ} \mathrm{C}$ \\
Pressure & $1 \mathrm{~Pa}$ \\
Power & $80 \mathrm{~W}$ \\
Thickness & $0.30 \mu \mathrm{m}$ \\
Postheat treatment & Quenching \\
\hline
\end{tabular}

As we know, PZT based material exhibits excellent piezoelectricity at Morphotropic Phase Boundary (MPB), and so the composition of $0.06 \mathrm{PMnN}-0.94 \mathrm{PZT}(45 / 55)$ we chose would own both high quality factor and high piezoelectricity because of its position around MPB.

\section{Results}

3.1. Crystal Structure. The structures of $0.06 \mathrm{PMnN}-$ 0.94PZT(45/55) thin film and PZT(45/55) thin film on $\mathrm{Si}(100)$ substrates were analyzed by XRD measurement, and the XRD spectra were shown in Figures 2(a) and 2(b), respectively.

It can be obtained that the 0.06PMnN-0.94PZT(45/55) thin film on $\mathrm{Si}(100)$ shows remarkable polycrystal structure with (111) and (101) orientations coexist, in which the (111) direction is the main orientation; however there are small peaks around the (101) orientation, and we analyzed that they are $\mathrm{Mn}_{3} \mathrm{O}_{4}$ and $\mathrm{PbO}$, respectively, which were marked in Figure 2(a). We believe that these two small compositions are attributed to the excess $\mathrm{MnO}_{2}$ and $\mathrm{PbO}$ in the target, and it is because we always add extra $10 \%$ mol percent $\mathrm{PbO}$ in the target, and the variable valence of $\mathrm{Mn}$ is another reason that there is $\mathrm{Mn}_{3} \mathrm{O}_{4}$ existing. We believe these two small peaks are very weak and nothing to affect the behaviors of $0.06 \mathrm{PMnN}-0.94 \mathrm{PZT}(45 / 55)$ thin films. For the nondoped PZT(45/55) thin films on $\mathrm{Si}(100)$, the XRD spectrum in Figure 2(b) shows that (111) and (101) orientations coexist similarly, and the (111) orientation is just a bit larger than (101) orientation. However, there is only one small peak of $\mathrm{PbO}$ near the (101) orientation, but it is stronger than 0.06PMnN$0.94 \mathrm{PZT}(45 / 55)$ thin film case which is because of the Mn and $\mathrm{Nb}$ substitution in PZT, and the $\mathrm{Mn}$ and $\mathrm{Nb}$ doping makes $\mathrm{Pb}$ more needed, and so there is Mn oxide excess. So the better choice for further research is to reduce the addition of $\mathrm{PbO}$. Whatever, the two XRD spectra show that the Mn and $\mathrm{Nb}$ doping and substitution are effective and reasonable, and both 0.06PMnN-0.94PZT(45/55) thin films and nondoped PZT(45/55) thin films show classical polycrystal structures with (111) and (101) orientations coexisting.

This result is distinctly different from the case with $\mathrm{MgO}(100)$ which shows the excellent single crystal structure of (001) orientation [8]. The important reason should be attributed to the crystal lattice match conditions between PMnN-PZT thin films and the substrates. Of course, we also can get pure (001) orientation PMnN-PZT thin film if we 


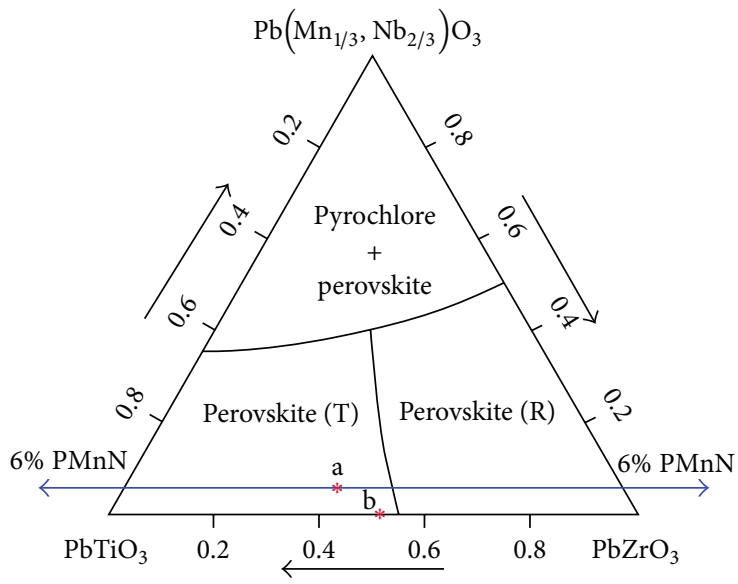

(a)

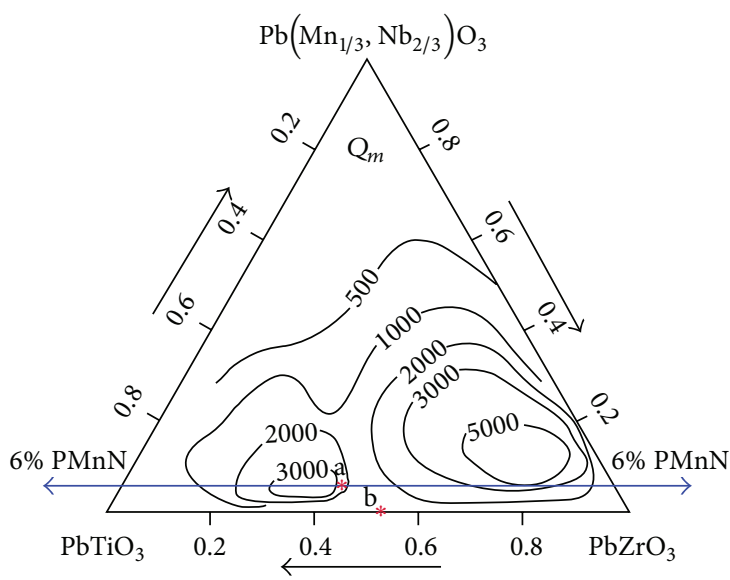

(b)

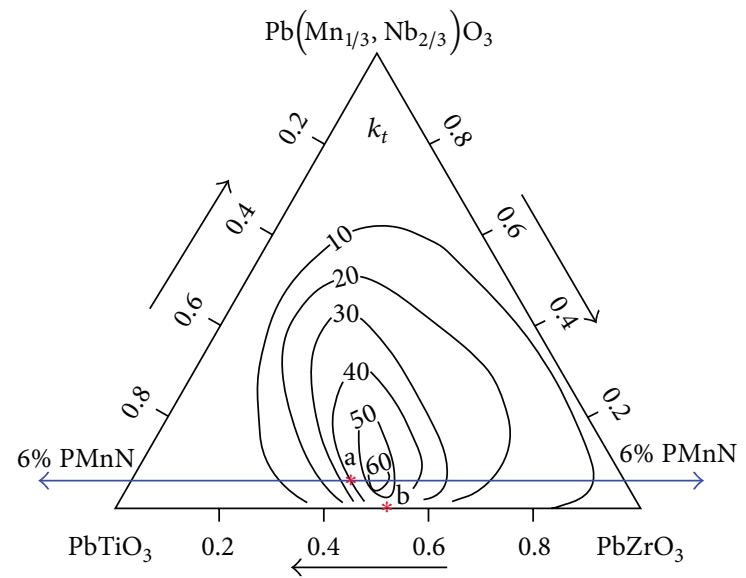

(c)

FIGURE 1: Material property with different compositions.

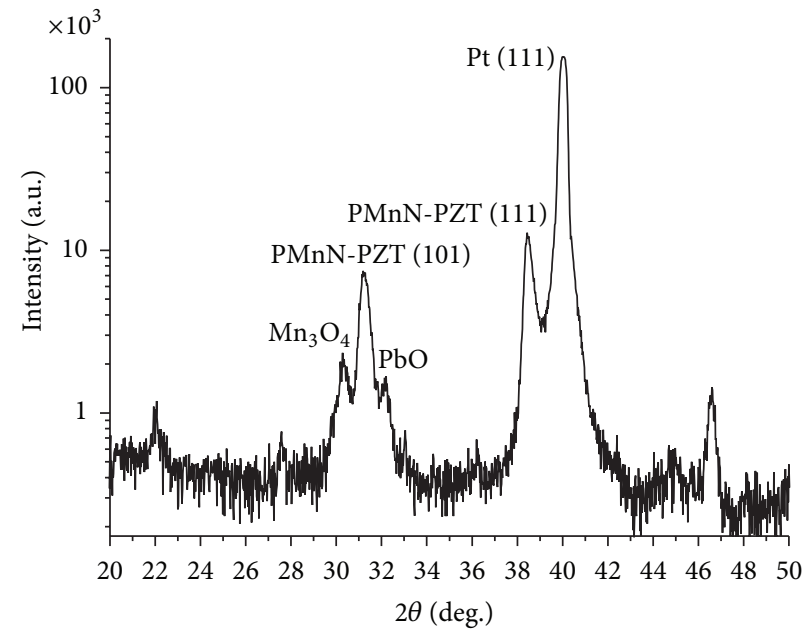

(a)

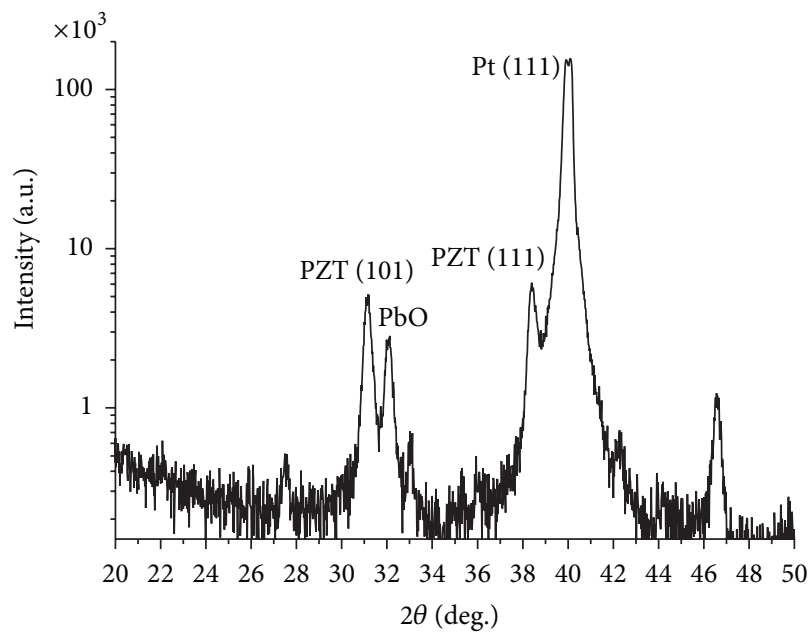

(b)

Figure 2: (a) XRD spectrum of 0.06PMnN-0.94PZT(45/55) thin film on Si substrate. (b) XRD spectrum of nondoped PZT(45/55) thin film on Si substrate. 


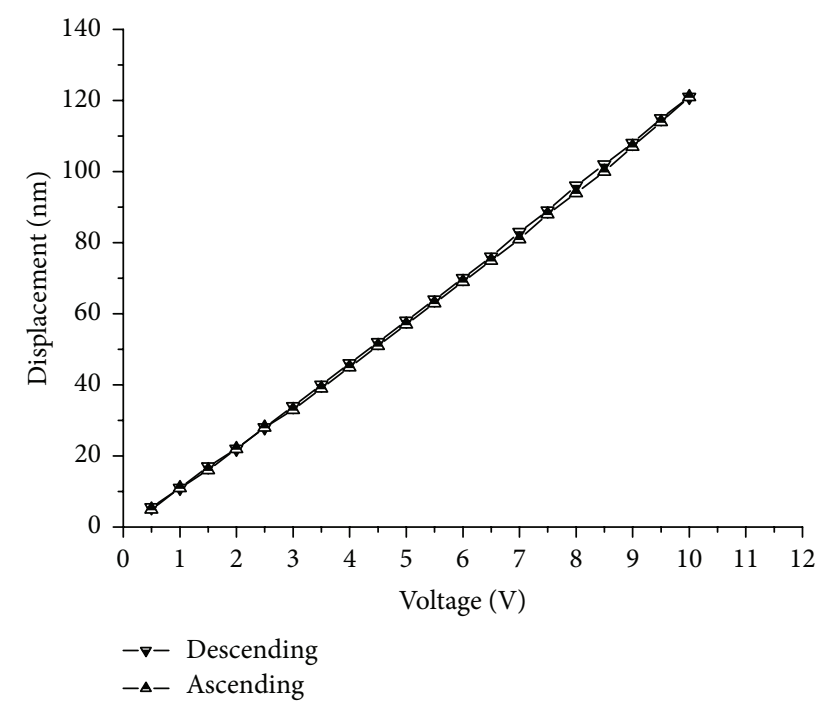

FIgURE 3: Vibration curve of 0.06PMnN-0.94PZT(45/55) thin film based cantilever.

make the match of crystal lattice perfect on silicon substrate using proper buffer layer on the substrate. However, it is not the necessary demand for the realistic applications of PMnNPZT thin film by costing so much effort and technique to do this.

3.2. Piezoelectricity. The transverse piezoelectric coefficient, $e_{31}$, was measured by the cantilever beams with the piezoelectric films covered by $(1)[8,9,12]$, and highly accurate laser

$$
{ }^{*} e_{31}=\frac{d_{31}}{s_{11}^{E}} \approx-\frac{\left(h^{s}\right)^{2}}{3 s_{11}^{s} L^{2} V} \delta
$$

vibrometer was used to test the displacement of vibration at $1 \mathrm{kHz}$ which is far from the cantilever beam resonance frequency of $4.4 \mathrm{kHz}$. In (1), $h^{s}, s_{11}^{s}$ are the thickness and the elastic compliance of the silicon substrates, respectively, $L$ is the length of the cantilever, $V$ is the applied voltage, and $\delta$ is the tip displacement of the beam under the voltage.

The measurement was made with both ascending voltage and descending voltage orders, and the displacement versus voltage curve of the PMnN-PZT film is shown in Figure 3, and the vibration is stable that the ascending curve is almost the same with that of descending one. Besides, both of the curves are linear enough that the inverse-piezoelectricity response is linear in the applied voltage range, and it also verifies that the $e_{31}$ measurement is reliable and accurate. Moreover, this kind of piezoelectric thin films can be used for piezoelectric device fabrications because of its excellent piezoelectric response behavior.

In this paper, $h^{s}=0.5 \mathrm{~mm}, L=9 \mathrm{~mm}, s_{11}^{s}=5.85 \times$ $10^{-12} \mathrm{~m}^{2} / \mathrm{N}, \delta=229.26 \mathrm{~nm}$, and $V=10 \mathrm{~V}$, then we get $e_{31}$ $=-4.03 \mathrm{C} / \mathrm{m}^{2}$ which is larger than the $e_{31}=-3.51 \mathrm{C} / \mathrm{m}^{2}$ of nondoped PZT(45/55) thin films on silicon substrates. However, these values are much smaller than $-7.7 \mathrm{C} / \mathrm{m}^{2}$ on $\mathrm{MgO}(100)$ case, and the polycrystal structure should be

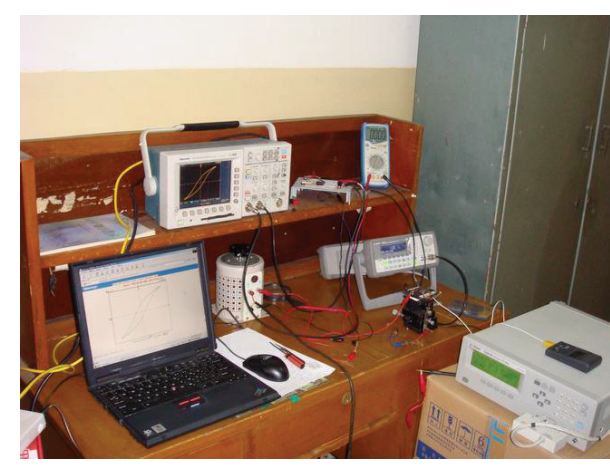

Figure 4: $P-E$ and dielectricity characterization system.

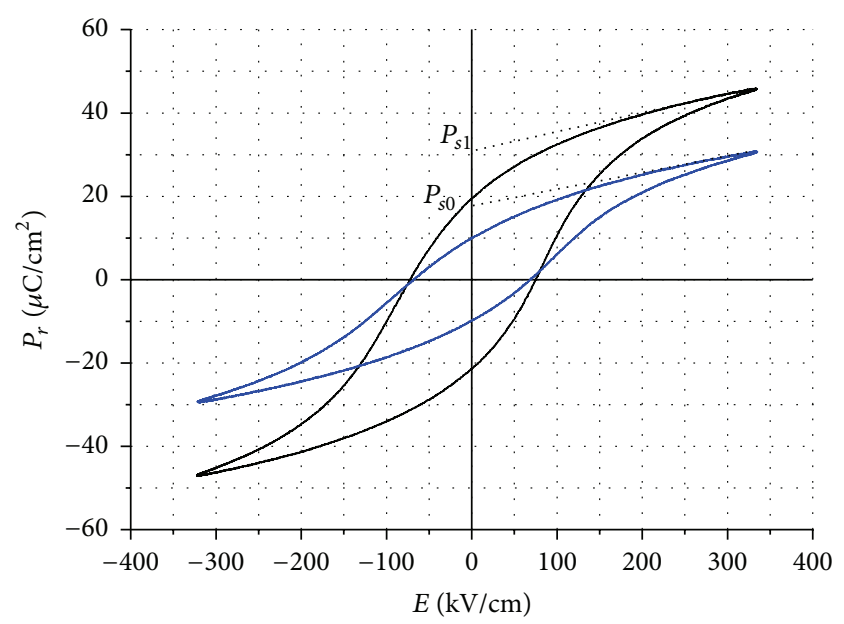

Figure 5: $P$ - E curves of $0.06 \mathrm{PMnN}-0.94 \mathrm{PZT}(45 / 55)$ thin film (black line) and nondoped PZT(45/55) thin film (blue line) on Si substrates.

the important reason for this, and it also can be obtained that we can improve the piezoelectricity by modifying the crystal lattice match for better single orientation. But it is acceptable comparing with better piezoelectricity, potentially high quality factor, and integration available.

3.3. Ferroelectricity. The ferroelectricity of $0.06 \mathrm{PMnN}$ $0.94 \mathrm{PZT}(45 / 55)$ thin films and nondoped PZT(45/55) thin films on silicon substrates were characterized with the $P-E$ curve by Sawyer-Tower circuit which was shown in Figure 4, and the hysteretic P-E curves were shown in Figure 5 respectively. We can get that the classical ferroelectricity exists for both these two kinds of thin films. The 0.06PMnN-0.94PZT(45/55) thin film owns distinctly better ferroelectric behavior than nondoped PZT(45/55) thin film case. In Figure 5, the $P_{s 1}=30.86 \mu \mathrm{C} / \mathrm{cm}^{2}$ and $P_{s 0}=17.74 \mu \mathrm{C} / \mathrm{cm}^{2}$ are the saturation polarization intensity of $0.06 \mathrm{PMnN}-0.94 \mathrm{PZT}(45 / 55)$ thin film and nondoped PZT(45/55) thin film on silicon substrates, respectively. The remnant polarization intensities are $P_{r}=20.44 \mu \mathrm{C} / \mathrm{cm}^{2}$ and $P_{r}=9.87 \mu \mathrm{C} / \mathrm{cm}^{2}$, respectively, and the coercive electric field intensities are $2 E_{c}=147.31 \mathrm{kV} / \mathrm{cm}$ and $2 E_{c}=135.44 \mathrm{kV} / \mathrm{cm}$ for $0.06 \mathrm{PMnN}-0.94 \mathrm{PZT}(45 / 55)$ thin film and PZT(45/55) 
thin film, respectively. We can see that the doped PZT(45/55) thin films, 0.06PMnN-0.94PZT(45/55) thin films, exhibit obviously better ferroelectric behavior than the nondoped PZT(45/55) thin films, such as the higher $P_{s}$, the higher $P_{r}$, and similar $2 E_{c}$, which shows that the PMnN doping in PZT(45/55) thin films shows obviously better improvements. The doped 0.06PMnN-0.94PZT(45/55) thin film also shows hard material behaviors than the nondoped PZT(45/55) thin film, and so we can judge that it has much higher quality factor than nondoped thin film.

There are small shifts in the $P-E$ curves on the electric field and polarization intensity for both kinds of thin films; however, these $P$-E curves show excellent symmetry, and so the reason of small shift of the $P$-E curve is the shift of ground electrode considering the symmetrical curve and the same linear shift of coercive electric field and polarization intensity.

These values are all smaller than those on $\mathrm{MgO}$ substrates with the $2 E_{c} \cong 230 \mathrm{kV} / \mathrm{cm}$ and the $P_{s} \cong 60 \mu \mathrm{C} / \mathrm{cm}^{2}$ [8], which can be described by the different crystal structures, and the 0.06PMnN-0.94PZT(45/55) thin films on MgO substrates show excellent hard material behaviors; however the films on Si substrates show the soft ferroelectric properties. The $P-E$ curve of the $0.06 \mathrm{PMnN}-0.94 \mathrm{PZT}(45 / 55)$ thin films on silicon substrates also shows that there are nonunity ferroelectric domains in the material. However, the different properties are attractive for different applications, such as the lower coercive electric field intensity, which makes the ferroelectric application lower voltage and energy needed.

3.4. Dielectricity. The dielectricity of $0.06 \mathrm{PMnN}-0.94 \mathrm{PZT}$ $(45 / 55)$ thin films and nondoped PZT(45/55) thin films on silicon substrates were measured by LCR meter which was also shown in Figure 4, and the relative dielectric constants $\varepsilon_{r}=681$ and $\varepsilon_{r}=537$, respectively, and the dielectric loss factors are $D=5 \%$ and $D=4.3 \%$, respectively. It can be obtained that $\mathrm{Mn}$ and $\mathrm{Nb}$ doping increases the dielectric constant and also makes the dielectric loss larger even though the change is small.

These results are distinctly different from the dielectric constant of 260 on $\mathrm{MgO}$ substrate, and they are almost two times of that on $\mathrm{MgO}$ substrate. It is reasonable for the different dielectric behaviors with different substrates, and the polycrystal structure of thin films on silicon substrate should be the main source of the main difference. However, the high dielectricity can be accepted for the micro device applications as well, especially considering the nondoped PZT(45/55) thin films on silicon substrates, which is also appreciated for the high power and high energy storage applications.

\section{Conclusions}

In this paper, the $0.06 \mathrm{PMnN}-0.94 \mathrm{PZT}(45 / 55)$ thin films and nondoped PZT(45/55) thin films on silicon substrates were deposited by magnetron sputtering method, and the properties were compared with each other and also compared with the same composition thin films on $\mathrm{MgO}$ substrates. The XRD results show that both $0.06 \mathrm{PMnN}-0.94 \mathrm{PZT}(45 / 55)$ thin films and nondoped PZT(45/55) thin films on silicon substrates exhibit classical polycrystal structures with (111) and (101) orientations coexisting, and the results show that the $\mathrm{Mn}$ and $\mathrm{Nb}$ doping and substitution in $\mathrm{PZT}(45 / 55)$ are effective even though they are not as significant as the corresponding single crystal thin films on $\mathrm{MgO}$ substrates.

The piezoelectric characterizations show $e_{31}=$ $-4.03 \mathrm{C} / \mathrm{m}^{2}$ and $e_{31}=-3.51 \mathrm{C} / \mathrm{m}^{2}$ for $0.06 \mathrm{PMnN}$ $0.94 \mathrm{PZT}(45 / 55)$ thin films and nondoped PZT(45/55) thin films, respectively, and it shows that the $\mathrm{Mn}$ and $\mathrm{Nb}$ doping improves the piezoelectricity of PZT(45/55) thin films. However, these values are smaller than the single crystal case of $\mathrm{MgO}$ substrate, but these values are acceptable for the applications, and particularly the remarkable linear piezoelectric response behavior of $0.06 \mathrm{PMnN}$ 0.94PZT(45/55) thin film is significant for the piezoelectric applications.

Moreover, the ferroelectric properties characterized by Sawyer-Tower Circuit show that both of the doped and nondoped PZT(45/55) thin films exhibit classical ferroelectricity. The 0.06PMnN-0.94PZT(45/55) thin film shows more obvious ferroelectric behaviors with higher saturation polarization, higher remnant polarization, and similar coercive electric field, and particularly the harder behavior of $0.06 \mathrm{PMnN}-0.94 \mathrm{PZT}(45 / 55)$ thin film exhibits the higher quality factor. But these values are smaller than the single crystal thin films on $\mathrm{MgO}$ substrates which own excellent hard ferroelectric material behavior; however, they are not all the bad results that the lower coercive electronic field is expected for the memory device applications with lower voltage and lower energy needed.

Finally, the dielectricity of two kinds of thin films was also studied, and the similarly high dielectric constants as the bulk PZT based materials were observed with $\varepsilon_{r}=681$ and $\varepsilon_{r}=537$ for the doped and nondoped PZT(45/55) thin films, respectively, and the dielectric loss of $D=5 \%$ and $D=4.3 \%$, respectively. The results show that $\mathrm{Mn}$ and $\mathrm{Nb}$ doping elevates the dielectricity of PZT(45/55) thin film and also rises the dielectric loss even though the change is small. The dielectric property of $0.06 \mathrm{PMnN}-0.94 \mathrm{PZT}(45 / 55)$ thin film is larger than that case of $\mathrm{MgO}$ substrate; however, it is still endurable. On the other hand, it is good choice for the high voltage and high energy storage applications.

In conclusion, we fabricated 0.06PMnN-0.94PZT(45/55) thin films and nondoped PZT(45/55) thin films on silicon substrates in this research and studied the crystal structures, the piezoelectricity, the ferroelectricity, and the dielectricity. Moreover we contrasted these properties among the doped and nondoped PZT(45/55) thin films on silicon substrates for the effects of $\mathrm{Mn}$ and $\mathrm{Nb}$ doping and also compared them with the single crystal structure 0.06PMnN-0.94PZT(45/55) thin films on $\mathrm{MgO}$ substrates. It can be obtained that $\mathrm{Mn}$ and $\mathrm{Nb}$ doping mostly improves the properties of crystal structure, piezoelectricity, ferroelectricity, and mechanical quality factor; of course it also makes the dielectricity and dielectric loss a bit larger. All the above research are significant for the research of ternary compound PZT based thin films research; particularly the successful fabrication of 0.06PMnN-0.94PZT(45/55) thin film on silicon substrates 
which are often used for the integration is very important for the $0.06 \mathrm{PMnN}-0.94 \mathrm{PZT}(45 / 55)$ realistic applications.

\section{Conflict of Interests}

The authors declare that there is no conflict of interests regarding the publication of this paper.

\section{Acknowledgments}

This work is supported by National Natural Science Foundation of China (no. 61201088, no. 11404257, no. 11504291, and no. 11304243), Shaanxi Province Innovation Project for Science and Technology Overall Planning (no. 2012KTCL0112), Industrialization Foundation of Shaanxi Educational Committee (no. 2011JG10), and Xi'an Technology Transferring and Promoting Project (CXY1342(5), CX1253(2)).

\section{References}

[1] P. Muralt, "PZT thin films for microsensors and actuators: where do we stand?" IEEE Transactions on Ultrasonics, Ferroelectrics, and Frequency Control, vol. 47, no. 4, pp. 903-915, 2000.

[2] P. B. Kirby, Q. X. Su, E. Komuro, Q. Zhang, M. Imura, and R. W. Whatmore, "PZT thin film bulk acoustic wave resonators and filters," in Proceedings of the IEEE International Frequency Control Symposium and PDA Exhibition, pp. 687-694, Seattle, Wash, USA, June 2001.

[3] P. Muralt, J. Antifakos, M. Cantoni, R. Lanz, and F. Martin, "Is there a better material for thin film BAW applications than A1N?" in Proceedings of the IEEE Ultrasonics Symposium, vol. 1, pp. 315-320, Rotterdam, The Netherlands, September 2005.

[4] T. Zhang, H. Zhang, Z.-Q. Wang, and S.-Y. Zhang, "Effects of electrodes on performance figures of thin film bulk acoustic resonators," Journal of the Acoustical Society of America, vol. 122, no. 3, pp. 1646-1651, 2007.

[5] Y. W. Yang, X. Shi, H. R. Lan et al., "Investigation on behavior of the vibration-based piezoelectric energy harvester array in ultracapacitor charging," Applied Physics Letters, vol. 106, no. 17, Article ID 173902, 2015.

[6] X.-H. Du, J. Zheng, U. Belegundu, and K. Uchino, "Crystal orientation dependence of piezoelectric properties of lead zirconate titanate near the morphotropic phase boundary," Applied Physics Letters, vol. 72, no. 19, pp. 2421-2423, 1998.

[7] D. Fu, H. Suzuki, T. Ogawa, and K. Ishikawa, "Highpiezoelectric behavior of $\mathrm{c}$-axis-oriented lead zirconate titanate thin films with composition near the morphotropic phase boundary," Applied Physics Letters, vol. 80, no. 19, pp. 3572-3574, 2002.

[8] T. Zhang, K. Wasa, I. Kanno, and S.-Y. Zhang, "Ferroelectric properties of $\mathrm{Pb}\left(\mathrm{Mn}_{1 / 3} \mathrm{Nb}_{2 / 3}\right) \mathrm{O} 3-\mathrm{Pb}(\mathrm{Zr}, \mathrm{Ti}) \mathrm{O}_{3}$ thin films epitaxially grown on (001)MgO substrates," Journal of Vacuum Science and Technology A: Vacuum, Surfaces and Films, vol. 26, no. 4, pp. 985-990, 2008.

[9] T. Zhang, S.-Y. Zhang, K. Wasa et al., "Effects of $\mathrm{Pb}(\mathrm{Mn}, \mathrm{Nb}) \mathrm{O}_{3}$ doping on the properties of PZT-based films deposited on silicon substrates," Physica Status Solidi (A), vol. 208, no. 10, pp. 2460-2466, 2011.

[10] Z. H. Duan, P. Chang, Z. G. Hu et al., "Temperature dependent Raman scattering and far-infrared reflectance spectra of $\mathrm{MgO}$ modified $\mathrm{Pb}_{0.99}\left(\mathrm{Zr}_{0.95} \mathrm{Ti}_{0.05}\right)_{0.98} \mathrm{Nb}_{0.02} \mathrm{O}_{3}$ ceramics: a composition effect," Journal of Applied Physics, vol. 116, Article ID 093513, 2014.

[11] P. Tirupathi, N. Kumar, M. Pastor, A. C. Pandey, and R. N. Choudhary, "Diffused phase transitions in $\mathrm{Pb}$ $\mathrm{Pb}\left(\mathrm{Zr}_{0.65} \mathrm{Ti}_{0.35}\right) \mathrm{O}_{3}-\mathrm{Pb}\left(\mathrm{Fe}_{2 / 3} \mathrm{~W}_{1 / 3}\right) \mathrm{O}_{3}$ multiferroics," Journal of Applied Physics, vol. 117, no. 7, Article ID 074105, 2015.

[12] I. Kanno, H. Kotera, and K. Wasa, "Measurement of transverse piezoelectric properties of PZT thin films," Sensors and Actuators A: Physical, vol. 107, no. 1, pp. 68-74, 2003. 

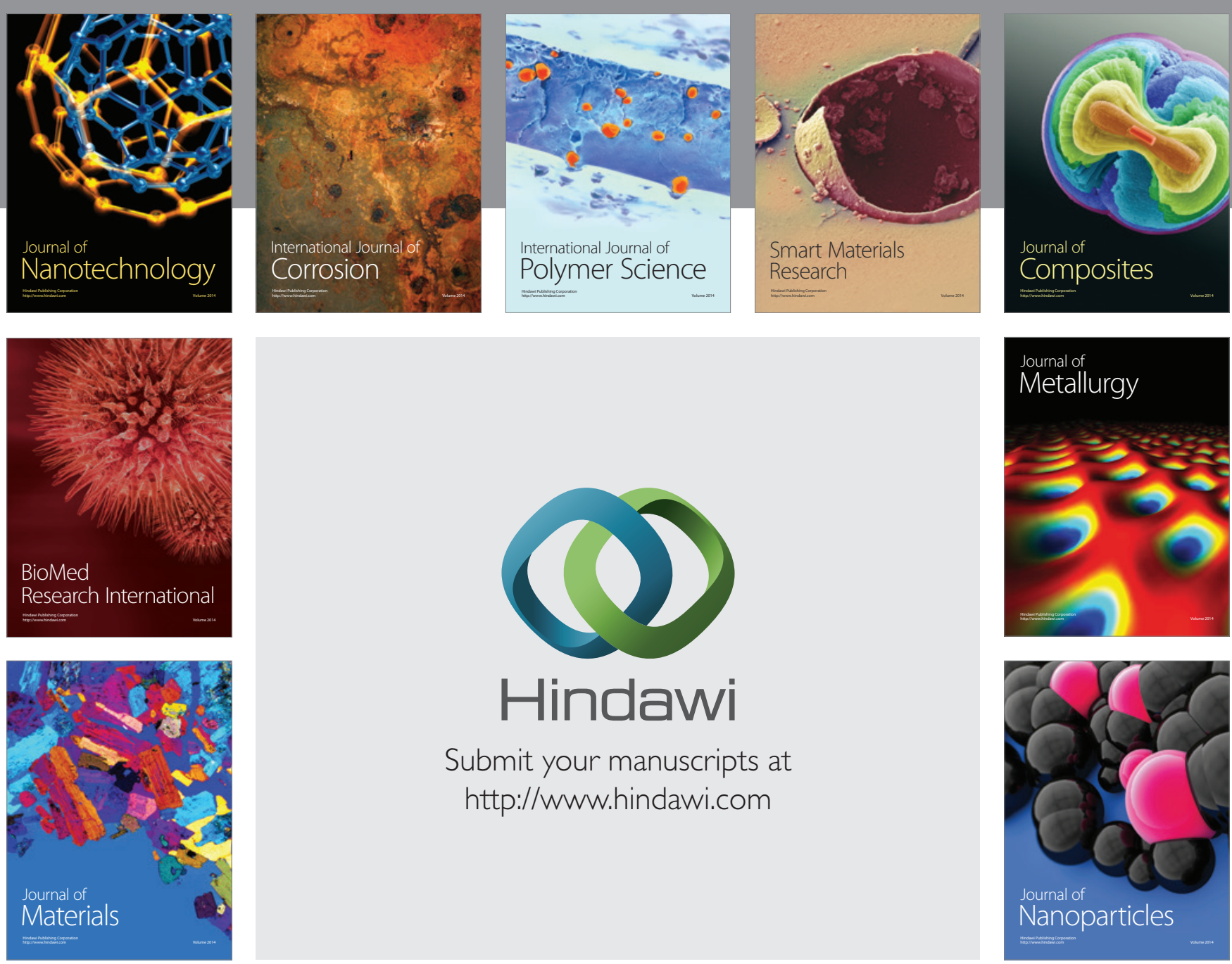

Submit your manuscripts at http://www.hindawi.com
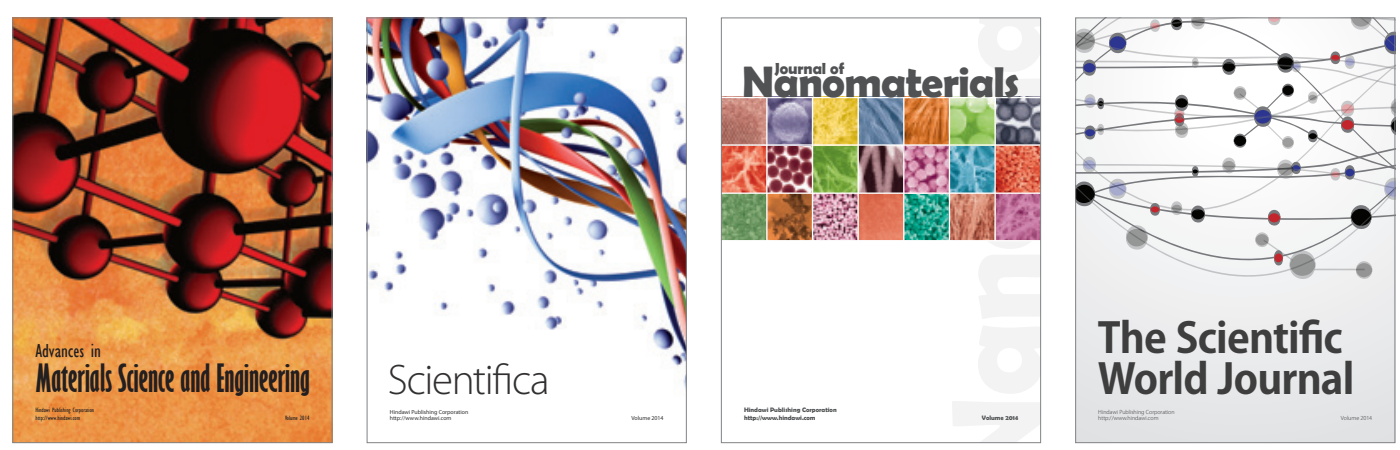

\section{The Scientific World Journal}
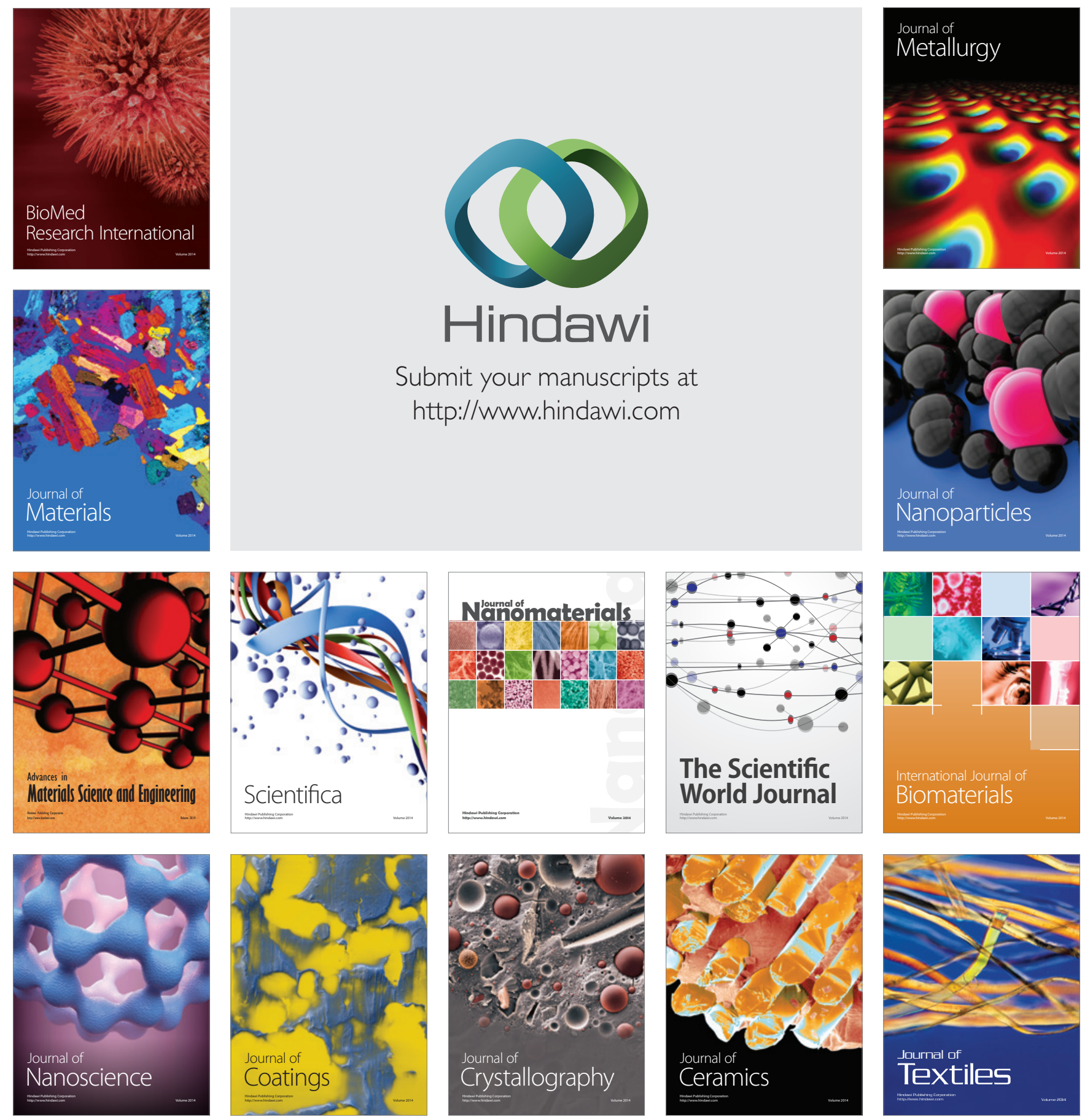